\title{
Induced membrane technique combined with antibiotic-loaded calcium sulfate-calcium phosphate composite as bone graft expander for the treatment of large infected bone defects: preliminary results of 12 cases
}

\author{
Zihou Zhao $^{1 \#}$, Guoliang Wang ${ }^{1 \#}$, Yong Zhang ${ }^{1 \#}$, Wen Luo ${ }^{2}$, Shiyu Liu ${ }^{3}$, Zhaohui Zeng ${ }^{1}$, Yunyan Liu ${ }^{1}$, \\ Yong Zhou ${ }^{1}$, Yunfei Zhang ${ }^{1}$ \\ ${ }^{1}$ Department of Orthopaedics, Second Affiliated Hospital, Air Force Medical University, Xi'an, China; ${ }^{2}$ Department of Ultrasound, Xijing Hospital, \\ Air Force Medical University, Xi'an, China; ${ }^{3}$ Institute of Oral Tissue Engineering, Air Force Medical University, Xi'an, China \\ Contributions: (I) Conception and design: Z Zhao, Y Zhang, S Liu; (II) Administrative support: Y Zhang, Y Zhou; (III) Provision of study materials \\ or patients: None; (IV) Collection and assembly of data: G Wang, Z Zeng, Y Liu; (V) Data analysis and interpretation: All authors; (VI) Manuscript \\ writing: All authors; (VII) Final approval of manuscript: All authors. \\ "These authors contributed equally to this work. \\ Correspondence to: Yunfei Zhang, PhD; Yong Zhou, PhD. Department of Orthopaedics, Second Affiliated Hospital, Air Force Medical University, \\ Xi’an, China. Email: tdbone@163.com; gukezy@fmmu.edu.cn.
}

Background: Management of large infected bone defects is a major clinical and socioeconomic problem. The induced membrane technique has been widely used as a solution. However, it has apparent disadvantages such as limited autologous bone graft supply and lack of continuous infection control. Meanwhile, calcium sulfate/calcium phosphate composites have efficacious osteogenesis and antibiotic delivery capacity. For the first time, we analyzed the efficiency of calcium sulfate/calcium phosphate composites as a bone graft expander in the induced membrane technique to treat large infected bone defects.

Methods: We retrospectively analyzed the clinical data of 12 patients with large infected bone defects of 6.1-17.2 cm treated with the induced membrane technique from November 2016 to July 2019. In the second reconstruction stage, the bone defect was filled with a mixture of the autogenous iliac bone and vancomycinimpregnated calcium sulfate/calcium phosphate composites at a ratio of 3:1. We assessed the bony union by Samantha X-ray score and recorded infection recurrence and complications. Paley scale and SF-36 score were used to evaluate the function of adjacent joint and quality of life pre and postoperatively. Pearson's correlation coefficients were calculated for union time and other clinical scores.

Results: The mean follow-up was 69 weeks (ranging from 30 to 142) after the second stage of the operation. The mean Samantha X-ray score was 5.1 [3-6], preoperative and postoperative SF-36 scores showed that there were statistical differences in all the nine aspects, and the excellent rate of adjacent joint function was $75 \%$ (Paley). All cases were radiologically healed, and none of the 12 patients had infection recurrence or failure of fixation at the last follow-up. Two cases had delayed wound healing and were cured after dress changing. There was a significant correlation between union time and Samantha X-ray score ( $\mathrm{r}=-0.887 ; \mathrm{P}=0.000)$, while there was no correlation between filling dose, size of the defect, and other outcomes.

Conclusions: This study provided evidence supporting calcium sulfate/calcium phosphate composites as an effective and safe bone graft expander in the induced membrane technique to treat large infected bone defect. This technique may help decrease the use of autologous bone graft and enhance the anti-infection effect of the induced membrane technique.

Keywords: Induced membrane technique; calcium phosphate (CP); calcium sulfate (CS); bone infections; bone substitutes; bone defects 
Submitted Feb 25, 2020. Accepted for publication Jul 19, 2020.

doi: 10.21037/atm-20-1932

View this article at: http://dx.doi.org/10.21037/atm-20-1932

\section{Introduction}

Critical-sized bone defects refer to those defects 2-2.5 times greater than the diameter of diaphysis that are too wide for self-restoration $(1,2)$. A large infected bone defect caused by chronic osteomyelitis is still a considerable challenge in bone reconstruction.

The induced membrane technique was first presented and applied in the clinic by Masquelet in 1986, and was used especially for the treatment of segmental bone defects. The strategy proceeds through the two following stages: stage 1 involves membrane induction by polymethyl methacrylate cement (PMMA) after thorough debridement, and stage 2 involves defect reconstruction with autologous cancellous bone or other artificial bone. Since its invention, it has been widely used in the field of orthopedics due to the operation being simple and providing outstanding results (3). The characteristics of the technique can be described as follows: Firstly, this technique is a model of multiple simultaneous osteogeneses rather than the traditional longitudinal creep model, and offers a rapid osteogenesis. Secondly, the induced membrane around bone cement not only has the function of local mechanical isolation, but also offers a stable circumstance for osteogenesis and assists in bone graft implantation (4). Finally, its abundant blood vessels allow it to produce cytokines like BMP and TGF- $\beta$, which provide strong support for the absorption, osteogenesis, and reconstruction of bone substitutes (4-6).

Surgeons' interest in this technique centers around the high demand for bone substitutes when filling large defects during stage 2 of the operation. Due to the limited availability of autologous bone, it is often impossible to fill large bone defects with autologous bone grafts alone, even though these carry no risk of immunological reaction or infection $(7,8)$. As an autologous bone substitute, allogeneic bone is a limited source and holds a potential risk of disease transmission (HIV or hepatitis $\mathrm{B}$ and $\mathrm{C}$ virus transmission) (9-11). Tissue-engineered bone grafting is a relatively new approach which has been applied as an alternative to conventional bone grafting, but its clinical application still needs exploration. Also, synthetic substitutes like the calcium sulfate/calcium phosphate (CS/CP) composite (75\% CS and $25 \% \mathrm{CP}$ ) have been proven to be an effective alternative for the treatment of bone defects (12). The CS/CP composite combines the benefits of both of its constituent materials and exhibits the properties of biocompatibility, bone induction, and osteoconduction (13-16).

Moreover, the combination of these two materials in the proper proportion provides an ideal resorption rate that enables the composite graft to keep pace with the rate of bone formation. Additionally, its microporous structure also enables satisfactory antibiotic loading and maintenance of high local antibiotic concentrations over extended periods. Furthermore, due to the introduction of $\mathrm{CP}$, the mechanical strength of the composite is superior to CS and autogenous cancellous bone (17). Both animal studies and clinical studies have demonstrated the satisfactory osteogenesis and antibiotic loading effect of CS/CP composites (18-20).

The value in our approach of combining vancomycinloaded CS/CP with autologous cancellous bone in induced membrane technology is that it overcomes limited sources of autogenous cancellous bone, thus enabling local antibiotic delivery and enhancing mechanical strength. Although CS/CP has been applied in various clinical fields like the treatment of necrosis of the femoral head, bone cysts, and bone tumors, no studies have analyzed the clinical effect of the induced membrane technique associated with $\mathrm{CS} / \mathrm{CP}$ as an autologous bone graft expander in the treatment of large infected bone defects $(18,19,21)$.

We applied CS/CP composite (Wright Medical Group, Memphis, TN, USA) to the induced membrane technique and evaluated the treatment performance. We aimed at (I) analyzing the bone reconstruction ability of the CS/CP composite as an autologous bone volume supplement and (II) evaluating the postoperative infection control and recovery of function provided by this technique. We present the following article following the STROBE reporting checklist (available at http://dx.doi.org/10.21037/atm-20-1932).

\section{Methods}

\section{Participants}

Twelve patients managed by the induced membrane technique between November 2016 and July 2019 were included in this retrospective study. There were 9 males and 
3 females with an average age of 39.5 years (18-59 years). The study was conducted at one tertiary level hospital, and the same surgeon performed all the procedures. The study was conducted in accordance with the Declaration of Helsinki (as revised in 2013). Our study received permission from the ethics committee of our institution and was performed with their guidance and help (No. TDLL-201709-19). All patients provided informed consent preoperatively.

\section{Inclusion criteria}

Patients with a large segmental infected bone defect of over $6 \mathrm{~cm}$ of long bone (humerus, femur, tibia) caused by trauma or osteomyelitis, who consented to admission for induced membrane technique were included in the study. Meanwhile, these patients had no fractures in the same limb as the defect and no nerve or major vascular injuries, and had good soft tissue conditions.

\section{Exclusion criteria}

The exclusion criteria were as follows: acute osteomyelitis with systemic symptoms, large bone defect with severe soft tissue and vascular loss, autologous bone deficiency caused by any reason, low immune function or acquired immune deficiency syndrome, diabetes or systemic malnutrition, allergy to CS or CP, severe cardiovascular diseases, and liver or kidney dysfunction.

The distribution of bone defects were as follows: one in the humerus, three in the femur, and eight in the tibia. Pathogenesis was as follows: three were post-traumatic, and nine were caused by infection after internal fixation. The wound secretions were cultured, and we found two cases of methicillin-sensitive Staphylococcus aureus (MSSA), two cases of methicillin-resistant Staphylococcus aureus (MRSA), one case of Enterobacter cloacae, and one case of Staphylococcus simulants, with six cases being negative, all of which were sensitive to vancomycin. The induced membrane technique combined with the antibiotic-loaded CS/CP composite as an autologous bone graft expander was applied to manage the large infected bone defect in the second stage of reconstruction. We mainly used bone union, recurrence of infection, and function as the outcome.

\section{Operative procedure}

Preoperative X-ray, computed tomography (CT), and occasional magnetic resonance imaging (MRI) scans were routinely performed to evaluate the scope of infection and to preliminarily calculate the number of autologous grafts required. We assessed the pelvis of each patients to determine the conditions of bone graft donor site. After discussion among senior physicians and with the patient's consent, the second reconstruction stage of induced membrane technique was scheduled. If the autologous bone proved to be insufficient, the CS/CP composite was applied. We selected the types of fixation according to the fracture site, including locking plate, intramedullary nail, external fixator, and Kirschner pin.

We paid considerable attention to the thoroughness of debridement and stability of the fracture. During stage 1 of the technique, the earlier fixations were removed if there were any. Then, a procedure to eliminate the sinus tract and complete debridement of the soft tissues was performed. Intraoperative frozen-section analysis was performed to clarify the scope of debridement by counting the number of neutrophils under a high magnification. A bone window was made at a predetermined position by using an electric drill and oscillating saw, after which, pus was removed from the intramedullary cavity. The medullary cavity was enlarged with a soft reaming drill to observe blood seeping into the bone window (capsicum sign positive). Necrotic bone was collected using a laminectomy rongeur, and the bone end was ground with osteotomes until bleeding was observed. Subsequently, the cavity was irrigated two or three times with sterile saline and hydrogen peroxide, after which it was again debrided. Later, the wound was soaked in dilute iodophor saline for 5 minutes, and the surgical drapes and gloves were replaced. Then, proper fixations were applied to stabilize the bone defect (If needed). PMMA cement (OSTEOPAL ${ }^{\circledR} \mathrm{V}$, Heraeus Medical GmbH, Germany) was prepared and placed into the defect after molding to induce membrane formation. We mixed $40 \mathrm{~g}$ PMMA with $5 \mathrm{~g}$ of vancomycin, as previously reported (22). During the polymerization of the PMMA cement, sterile saline was continuously used to reduce damage to the surrounding soft tissues caused by the heat generated by the polymerization. If possible, primary closure was suggested, and if not, a flap transplant was applied. Postoperatively, we routinely used intravenous antibiotic therapy by drug sensitivity.

Oral and intravenous antibiotics were given postoperatively for 2 to 4 weeks. X-rays and CT scans were used to observe fracture alignment and the stability of internal fixation. Next, laboratory tests (white blood cell count, erythrocyte sedimentation rate, C-reactive protein) 

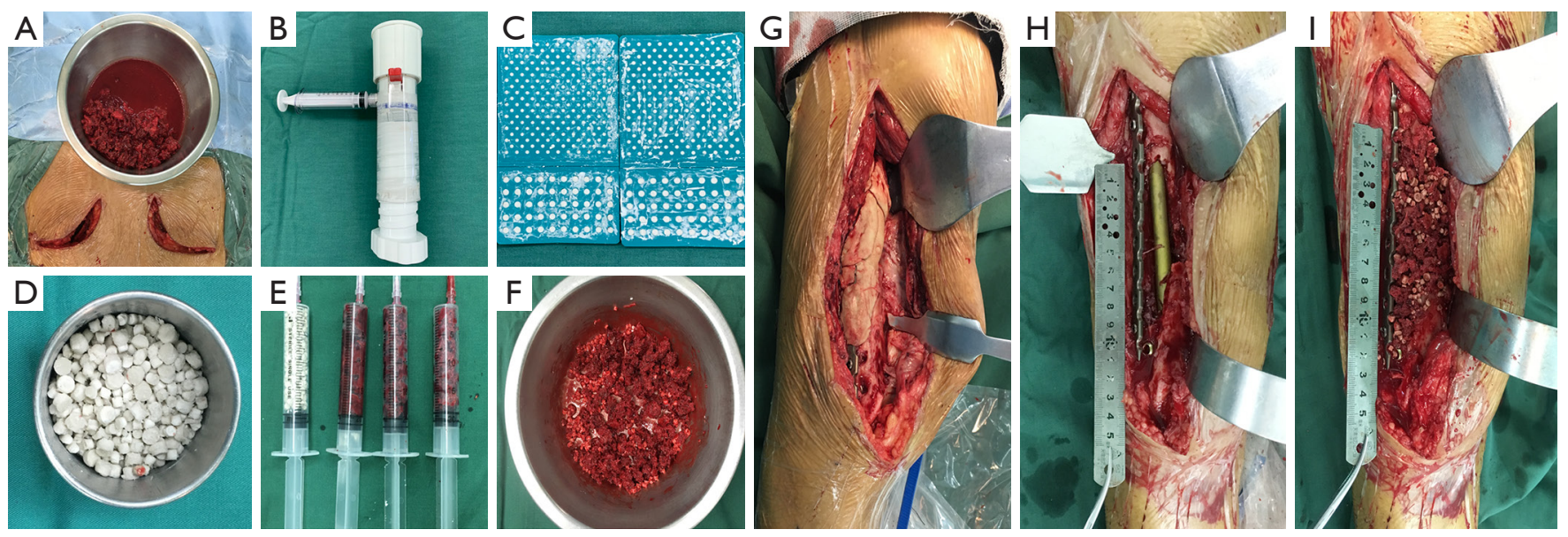

Figure 1 Patient with large infected bone defect of femur treated by CS/CP. (A) We obtained sufficient autogenous iliac bone after estimating the size of the defect, (B,C,D) prepared the CS/CP composite, and (E,F) then mixed them in a 3:1 ratio. (G) The membrane induced by the first stage of surgery was exposed by removing the PMMA cement while attention was paid to protect the membrane. (H) A bone defect size of about $10 \mathrm{~cm}$ was observed, and (I) the mixture was implanted into the defect, leaving no cavity. CS/CP, calcium sulfate/ calcium phosphate; PMMA, polymethyl methacrylate cement.

were conducted to record infection indicators before the second stage operation.

The second stage of the surgery was performed at least 2 months after stage 1 of the operation and consisted of reconstruction grafting using autogenous iliac bone and the CS/CP composite (Figure 1A,B,C,D,E,F). Briefly, an incision was made along the original surgical scar. A fully formed membrane induced by the PMMA cement was observed (Figure 1G). The membrane was carefully dissected and turned upward, after which the cement and plates were removed. The soft tissue was attached to the outside of the membrane, so it could not be easily damaged. Necrotic bone was taken until a positive capsicum sign was obtained (Figure 1H). We ensured that the proximal and distal medullary cavities were unobstructed. The wound was thoroughly irrigated two or three times with sterile saline and then soaked in a diluted iodine solution for 5 minutes. The size of the defect was exactly measured to calculate the volume of bone graft required. The $\mathrm{CS} / \mathrm{CP}$ composites were loaded with vancomycin by a concentration of $300 \mathrm{mg} / \mathrm{cc}$. Then, a 5 -cm-long curved incision was made at the highest point of right/left posterior superior iliac spine, and a $3 \times 2 \mathrm{~cm}^{2}$ iliac bone was harvested and cut into pellets with a rongeur. Some cancellous bone was scraped from the medullary cavity, and the bone marrow was extracted for later use. The mixed graft combined with autologous cancellous bone and the CS/CP composite at a ratio of
3:1 was then filled into the defect (Figure 1I), and internal fixation or external fixation was applied if needed. Patients were allowed to exercise without bearing weight 1 week postoperatively. Bone union was judged by radiological examination and was defined as $3 / 4$ cortices showing callus formation.

Functional exercise was guided postoperatively by professional physicians. Partial weight bearing could be permitted earlier if indicated by radiological examination. However, total weight bearing was not allowed until satisfactory corticalization was present.

\section{Data collection and assessment}

Patients were required to have follow-up visits in the clinic at 1, 3, 6, and 12 months after surgery. Baseline data including age, gender, site, etiology, fixation method, and size of the defect were collected. Complications and recurrence were recorded to evaluate the prognosis after treatment. Meanwhile, laboratory examinations, including white blood cell (WBC) count, C-reactive protein (CRP), and erythrocyte-sedimentation rate (ESR), were conducted and analyzed pre and postoperatively.

$\mathrm{X}$-rays were used to assess the quality of union with two independent observers using the Samantha radiographic grading scale to quantify the healing $(23,24)$ (Table 1$)$.

The Paley (25) scale was used to evaluate clinical 
Table 1 Radiographic grading scale for the degree of healing

\begin{tabular}{l}
\hline Grade description \\
\hline - No changes from the immediate postoperative appearance \\
- A slight increase in radiodensity distinguishable from the graft \\
- Recognizable increase in radiodensity, bridging of one cortex with new-bone formation to the graft \\
- Bridging of at least one cortex with the material of nonuniform radiodensity, early incorporation of the graft suggested by \\
the obscurity of graft borders \\
- Defect bridged on both medial and lateral sides with the bone with uniform radiodensity, cut ends of the cortex still visible, \\
and graft and new bone not easy to differentiate \\
- Same as grade 3, with at least one of four cortices obscured by new bone \\
- Defect bridged by new uniform bone, cut ends of cortex no longer distinguishable, and graft no longer visible
\end{tabular}

outcomes, including bone results and joint function. Four criteria were included to assess bone results: union, infection, deformity, and leg-length discrepancy. Excellent was defined as a bone union, no infection, deformity of less than $7^{\circ}$, and length discrepancy of less than $2.5 \mathrm{~cm}$. Good was defined as a union with the presence of any other two items. Fair was defined as a union with the presence any other single item. Poor was defined as nonunion or refracture.

Function was evaluated using the following 5 indicators: (I) pain; (II) significant limp; (III) soft tissue dystrophy; (IV) rigid contracture of surrounding joints; $(\mathrm{V})$ participation in normal daily activities. Excellent was defined as indicator 5 with no presence of the other four indicators. Good was defined as indicator 5 with the presence of half of the other four indicators. Fair was defined as indicator 5 with the presence of three of the four other indicators or an amputation. Poor was defined as being unable to perform routine daily activities.

The SF-36 scale was used to assess patients' quality of life from the following eight aspects: physical functioning, role-physical, bodily pain, general health, vitality, social functioning, role-emotional, and mental health (26). The results of preoperative and final follow-up were used to compare and analyze the statistical differences.

\section{Statistics analysis}

Data were collected and recorded with Microsoft Excel (2017). The quantitative data were presented as $\bar{x} \pm s$. We conducted the homogeneity test of variance for each group of data for preoperative and postoperative SF-36 scores, and then an independent $t$-test was applied. We used one- way analysis of variance (ANOVA) to examine the statistical difference of WBC and CRP between the groups and nonparametric tests to examine the statistical difference of ESR. Pearson's correlation coefficients were calculated for different size of defect and change in clinical scores. All calculations were performed using SPSS version 23.0. A P value $<0.05$ was considered statistically significant.

\section{Results}

Twelve patients with an average age of 39.5 years (1859 years) with large bone defects were included in this retrospective study. The mean duration in the hospital for stage 2 of the reconstruction operation was 27 days. Patients had a mean follow-up time of 69 weeks (30142 weeks). The mean times of operation in other medical institutions before admission to hospital was 1.5 . The mean size of the bone defect was $10 \mathrm{~cm}(6.1-17.2 \mathrm{~cm})$. None of the 12 patients had infection recurrence or wound-related problems. The membrane was successfully induced in all cases after stage 1 surgery. Preoperative mean ESR, CRP, and $W B C$ were $22.25 \mathrm{~mm} / \mathrm{h}, 3.56 \mathrm{mg} / \mathrm{L}$, and $6.56 \times 10^{9} / \mathrm{L}$, respectively. Patients' baseline data are provided in Table 2 .

During surgery of stage 2 , all bone defects were filled with autogenous iliac and CS/CP composite as a volume expander at a ratio of $3: 1$. The mean dose of CS/CP composite and the antibiotic load was $15 \mathrm{cc}$ mixed with vancomycin $(300 \mathrm{mg} / \mathrm{cc})$. Microbial culture showed that the most common bacteria were Staphylococcus (5 out of 12), all of which were sensitive to vancomycin. Ten patients received internal fixation or external fixator to support limb stability, and all remained stable without loosening or infection during follow-up. Two cases (two in the tibia) 
Table 2 Baseline and follow-up data of 12 cases

\begin{tabular}{|c|c|c|c|c|c|c|c|c|c|c|}
\hline Case & $\begin{array}{c}\text { Gender } \\
(\mathrm{M} / \mathrm{F})\end{array}$ & Age & Site & Pathogenesis & $\begin{array}{l}\text { Time of } \\
\text { surgery }\end{array}$ & Graft material & $\begin{array}{c}\text { Size of } \\
\text { defect }(\mathrm{cm})\end{array}$ & $\begin{array}{l}\text { CS/CP filling } \\
\text { dose (cc) }\end{array}$ & $\begin{array}{l}\text { Hospital } \\
\text { stay(days) }\end{array}$ & $\begin{array}{c}\text { Follow-up } \\
\text { (weeks) }\end{array}$ \\
\hline 1 & $\mathrm{M}$ & 30 & Tibia & Internal fixation & 2 & Iliac bone + CS/CP & 13.9 & 20 & 41 & 105 \\
\hline 2 & $\mathrm{~F}$ & 59 & Humerus & Internal fixation & 3 & Iliac bone + CS/CP & 17.2 & 19 & 22 & 98 \\
\hline 3 & M & 56 & Tibia & Internal fixation & 2 & Iliac bone + CS/CP & 12 & 21 & 31 & 142 \\
\hline 6 & $M$ & 56 & Tibia & Post-traumatic & 1 & lliac bone + CS/CP & 8 & 15 & 23 & 55 \\
\hline 7 & $\mathrm{~F}$ & 33 & Femur & Internal fixation & 2 & lliac bone + CS/CP & 9.9 & 12 & 20 & 57 \\
\hline 8 & M & 44 & Tibia & Post-traumatic & 0 & lliac bone + CS/CP & 6.2 & 5 & 25 & 47 \\
\hline 12 & $M$ & 33 & Femur & Internal fixation & 2 & lliac bone + CS/CP & 9 & 11 & 23 & 30 \\
\hline
\end{tabular}

Table 3 Preoperative and postoperative infection-related indicators $(\bar{x} \pm s)$

\begin{tabular}{lcccc}
\hline Item & Before stage 1 & Before stage 2 & After stage 2 & P \\
\hline WBC (/L) & $6.56 \pm 1.40$ & $6.83 \pm 1.32$ & $7.45 \pm 1.68$ & $0.333^{*}$ \\
ESR (mm/h) & $22.25 \pm 22.70$ & $7.83 \pm 3.56$ & $9.17 \pm 4.73$ & $0.079^{\$}$ \\
CRP (mg/L) & $3.56 \pm 2.26$ & $3.72 \pm 2.11$ & $3.72 \pm 2.08$ & $0.979^{*}$ \\
\hline
\end{tabular}

*, one-way ANOVA; ${ }^{\$}$, non-parametric test. WBC, white blood cell; ESR, erythrocyte-sedimentation rate; CRP, C-reactive protein.

had delayed wound healing and were cured after dress changing by using diluted iodophor saline (the results of bacterial culture were negative) with no other treatment. Primary healing of incisions was acquired in the rest of the patients, and infection was effectively eradicated during the follow-up. The mean ESR, CRP, and WBC 2 months after operation were $9.17 \mathrm{~mm} / \mathrm{h}, 3.72 \mathrm{mg} / \mathrm{L}$, and $7.45 \times$ $10 \% / \mathrm{L}$, respectively, which were all in the normal range. We conducted statistical analysis on the infection indicator (ESR, CRP, and WBC) before stage 1, before stage 2, and after stage 2, and found no significant difference between the groups (Table 3). Systemic antibiotic therapy (including intravenous and oral methods) lasted until 2-4 weeks after the second stage of operation and was adjusted according to the drug sensitivity results.

Six months after surgery, radiological examination showed that the bone defect had been filled by new bone with uniform density and that the osteotomy line was blurred. At around 3 months, primitive callus formation was apparent, and bone substitute absorption could be observed (Figures 2,3). The mean score of Samantha X-ray was 5.1 [3-6]. The mean progression to union time of the 12 patients was 29 weeks (16-48 weeks) (Table 4, Figures 2,3).

The union result assessed by the Paley scale showed excellent in 12 cases. Radiographs showed the presence of bone union and that no infection had occurred during follow-up. Additionally, all cases had achieved anatomical reduction with no deformity beyond $7^{\circ}$ and no length discrepancy of more than $2.5 \mathrm{~cm}$ occurring. In joint function assessment, nine cases achieved excellent, two achieved good, and one achieved fair. The excellent rate was $75 \%(9$ in 12). One case suffered from dysfunction of adjacent joints due to insufficient postoperative rehabilitation training (Table 3).

We compared the preoperative and postoperative SF36 scores, and the results showed that there were statistical 

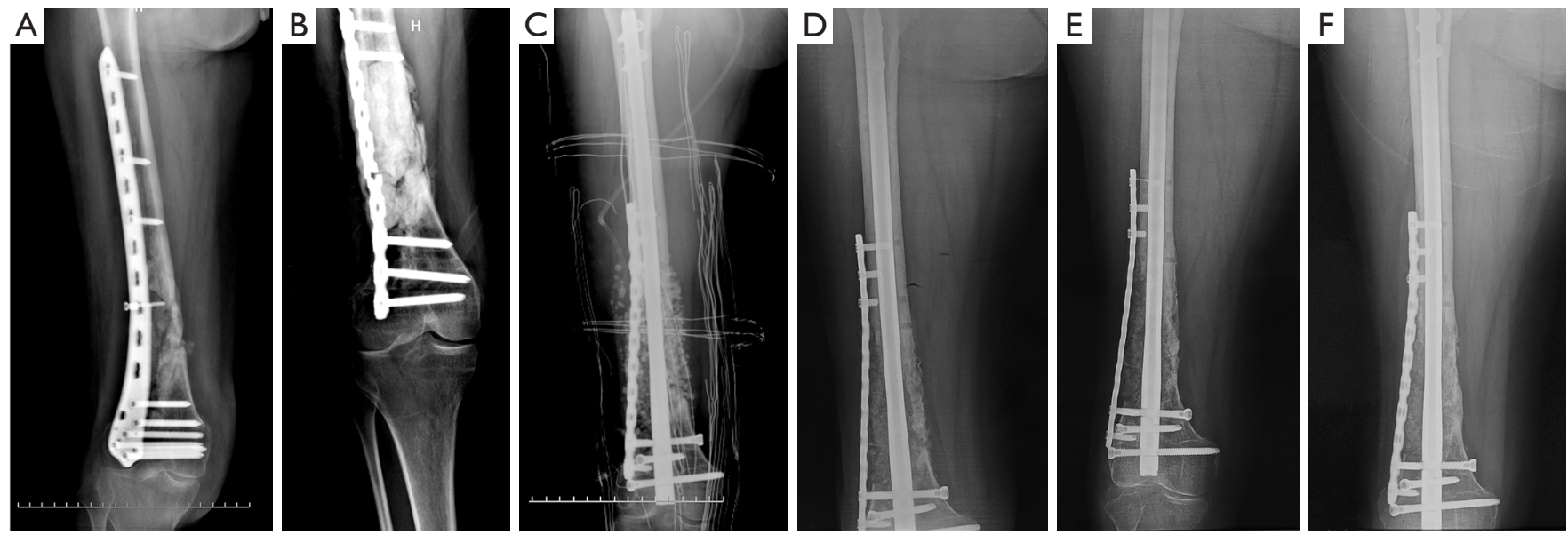

Figure 2 Typical radiographs of chronic osteomyelitis of femur treated with CS/CP (A) preoperatively, (B) managed with vancomycinloaded PMMA, locking plate. (C) Filling the bone defect with a mixture of autogenous iliac and vancomycin-impregnated CS/CP composite in a 3:1 ratio after thorough debridement. Follow-up at 3 (D), 6 (E), and 9 months (F). CS/CP, calcium sulfate/calcium phosphate; PMMA, polymethyl methacrylate cement.
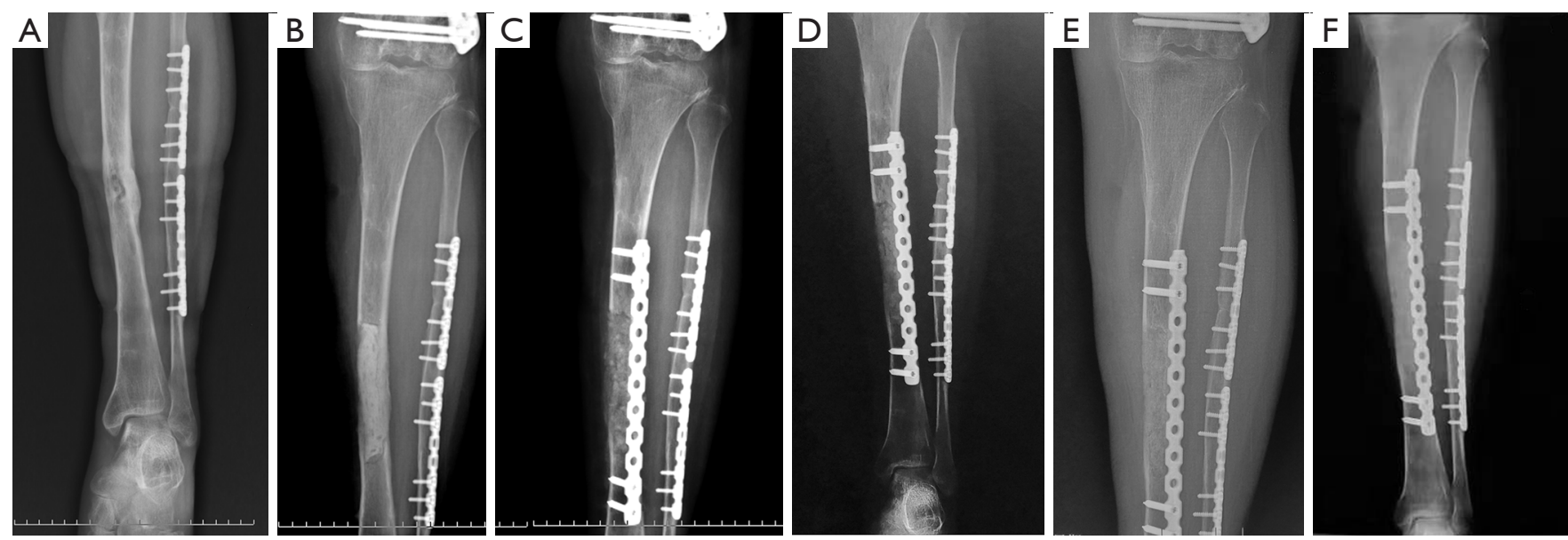

Figure 3 Typical radiographs of chronic osteomyelitis of the tibia treated with CS/CP (A) preoperatively, (B) managed with vancomycinloaded PMMA. (C) Filling the bone defect with a mixture of autogenous iliac and vancomycin-impregnated CS/CP composite after thorough debridement. (D) Follow up at 3, (E) 6, and (F) 9 months. CS/CP, calcium sulfate/calcium phosphate; PMMA, polymethyl methacrylate cement.

differences in all nine aspects $(\mathrm{P}<0.05)$ indicating that the postoperative quality of life of patients had dramatically improved (Table 5).

We analyzed the correlations between defect size, filling dose, union time, Samantha X-ray score, and other clinical outcomes. There was a significant correlation between union time and Samantha $\mathrm{X}$-ray score $(\mathrm{r}=-0.887 ; \mathrm{P}=0.000)$, while there was no correlation between defect size, filling dose, and other clinical outcomes.

\section{Case presentation}

A 58-year-old woman presented to our hospital with a 17 -year history of right humeral infected bone defect associated with right upper limb malformation, sinus shortening, and yellowish discharge (Figure 4A). During stage 1 of the induced membrane technique, necrotic and infected bone and soft tissues were thoroughly debrided. Then, one locking plate (SDDZ36, Medtronic Kanghui 
Table 4 Postoperative data analysis and results of 12 cases

\begin{tabular}{|c|c|c|c|c|c|c|c|c|}
\hline Case & Microbial culture & Fixation Method & $\begin{array}{l}\text { Samantha } \\
\text { X-ray score }\end{array}$ & $\begin{array}{l}\text { Paley bone } \\
\text { result }\end{array}$ & $\begin{array}{c}\text { Paley } \\
\text { functional } \\
\text { result }\end{array}$ & $\begin{array}{l}\text { Union time } \\
\text { (weeks) }\end{array}$ & Complications & Recurrence \\
\hline 2 & MSSA & 2 locking plate +3 screws & 3 & Excellent & Good & 48 & None & No \\
\hline 3 & Negative & $\begin{array}{l}\text { Intramedullary nail + } \\
4 \text { screws }\end{array}$ & 6 & Excellent & Excellent & 16 & $\begin{array}{l}\text { Sterile wound } \\
\text { leakage }\end{array}$ & No \\
\hline 4 & $\begin{array}{l}\text { Enterobacter } \\
\text { cloacae }\end{array}$ & None & 5 & Excellent & Excellent & 24 & $\begin{array}{l}\text { Sterile wound } \\
\text { leakage }\end{array}$ & No \\
\hline 5 & $\begin{array}{l}\text { Staphylococcus } \\
\text { simulans }\end{array}$ & 2 locking plate +4 screws & 6 & Excellent & Excellent & 23 & None & No \\
\hline 6 & Negative & $\begin{array}{l}\text { Intramedullary nail + } \\
3 \text { screws }\end{array}$ & 5 & Excellent & Good & 30 & None & No \\
\hline 8 & Negative & $\begin{array}{l}\text { External fixator }+1 \text { locking } \\
\text { plate }+6 \text { screws }\end{array}$ & 4 & Excellent & Fair & 36 & None & No \\
\hline 9 & MSSA & $\begin{array}{l}\text { External fixator }+1 \text { locking } \\
\text { plate }+6 \text { screws }\end{array}$ & 6 & Excellent & Excellent & 28 & None & No \\
\hline 10 & MRSA & 1 locking plate +10 screws & 5 & Excellent & Excellent & 29 & None & No \\
\hline 11 & MRSA & $\begin{array}{c}1 \text { locking plate }+8 \text { screws }+ \\
\text { Intramedullary nail }\end{array}$ & 5 & Excellent & Excellent & 30 & None & No \\
\hline 12 & Negative & $\begin{array}{c}1 \text { locking plate }+9 \text { screws }+ \\
\text { Intramedullary nail }\end{array}$ & 5 & Excellent & Excellent & 26 & None & No \\
\hline
\end{tabular}

MSSA, methicillin-sensitive Staphylococcus aureus.

Table 5 Preoperative and postoperative SF-36 scale scores $(\bar{x} \pm s)$

\begin{tabular}{lccc}
\hline Classification & Pre-op SF-36 score & Post-op SF-36 score & P \\
\hline Bodily pain & $51.50 \pm 9.80$ & $64.50 \pm 7.59$ & $0.001^{*}$ \\
Physical functioning & $21.67 \pm 9.61$ & $63.33 \pm 9.85$ & $0.000^{*}$ \\
Role-physical & $47.92 \pm 16.71$ & $70.83 \pm 25.75$ & $0.017^{*}$ \\
General health & $31.67 \pm 10.52$ & $57.50 \pm 10.47$ & $0.000^{*}$ \\
Vitality & $38.75 \pm 6.44$ & $60.83 \pm 9.49$ & $0.000^{*}$ \\
Social functioning & $42.59 \pm 19.44$ & $61.11 \pm 13.81$ & $0.013^{*}$ \\
Role-emotional & $30.56 \pm 26.43$ & $86.11 \pm 17.16$ & $0.000^{*}$ \\
Mental health & $50.00 \pm 7.14$ & $67.00 \pm 8.20$ & $0.000^{*}$ \\
Reported health transition & $37.50 \pm 13.06$ & $60.42 \pm 12.87$ & $0.000^{*}$ \\
\hline
\end{tabular}

*, $\mathrm{P}<0.05$. 

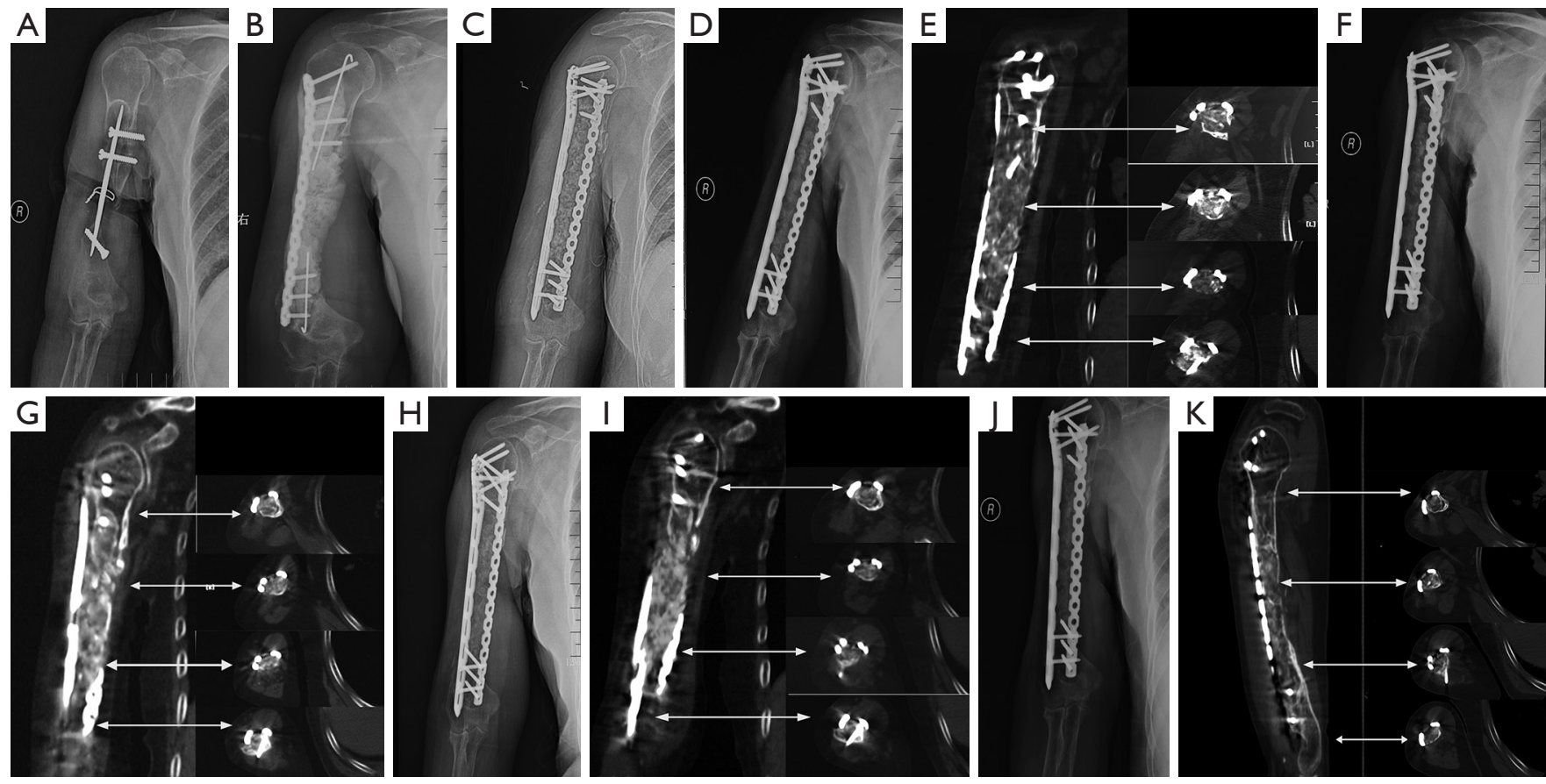

Figure 4 Typical X-ray and CT scans of chronic osteomyelitis of humerus treated with CS/CP. (A) Plain radiographs before the first stage of surgery showed a large bone defect. (B) PMMA was implanted in the first stage. (C) X-ray examination immediately after the second stage of surgery showed that the bone defect was completely filled with the calcium sulfate/calcium phosphate composites and autologous bone. (D,E,F,G,H,I,J,K) Plain radiographs and CT scans of the bone defect taken at (D,E) 4 months, (F,G) 6 months, (H,I) 9 months, and (J,K) 12 months postoperatively. With the formation of new bone, the composite was gradually absorbed, no new defects formed until new bone formation was complete, and the rate of absorption was comparable to new bone formation. (J,K) At 12 months after surgery, the composite was almost completely absorbed, and new bone formation was complete which was equivalent to the density of the surrounding bone. CS/ CP, calcium sulfate/calcium phosphate; PMMA, polymethyl methacrylate cement.

Orthopedics, Changzhou, China) and screws (SDLD03, Medtronic Kanghui Orthopedics, Changzhou, China) were used to fix the bone defect. PMMA cement was placed into the defect after molding to induce membrane formation (Figure 4B). Two weeks after the operation, shoulder and elbow-joint mobilization were encouraged, and partial loading was allowed and gradually increased.

The second stage of the induced membrane technique surgery was delayed and performed 8 months after the implantation of PMMA cement and consisted of reconstruction grafting using autogenous iliac bone and the CS/CP composite at a ratio of 3 to 1 (Figure $4 C$ ). The size of the defect was measured as about $78 \mathrm{cc}$ with a length of $17.2 \mathrm{~cm}$. Afterward, $58 \mathrm{cc}$ autologous iliac plate was harvested and cut into pellets from the left and right posterior superior iliac spine. Some cancellous bone was scraped from the medullary cavity. As the volume accounted for only three-quarters of the defect, we prepared $19 \mathrm{cc}$ of the CS/CP composite material loaded with vancomycin $(300 \mathrm{mg} / \mathrm{cc})$ according to the manufacturer's instructions (Wright Medical Group, Memphis, TN, USA). We fixed the humerus with one PHILOS plate and one locking plate and screws (DePuy Synthes Company, Somerville, NJ, USA).

Four, six, and nine months after the second stage of induced membrane technique surgery, the internal fixation was stable (Figure 4D,E,F,G,H,I), and the patient could carry out simple daily activities like combing hair, pouring water, and sweeping the floor. The latest X-ray and CT scans, performed 12 months after the second surgery, showed that about $90 \%$ of the CS/CP composite material had been absorbed, and bone continuity had been restored (Figure 47,K). Single-photon emission CT/computerized tomography (ECT/CT) scans performed at 9 months revealed active bone metabolism, suggesting new bone formation (Figure 5). 


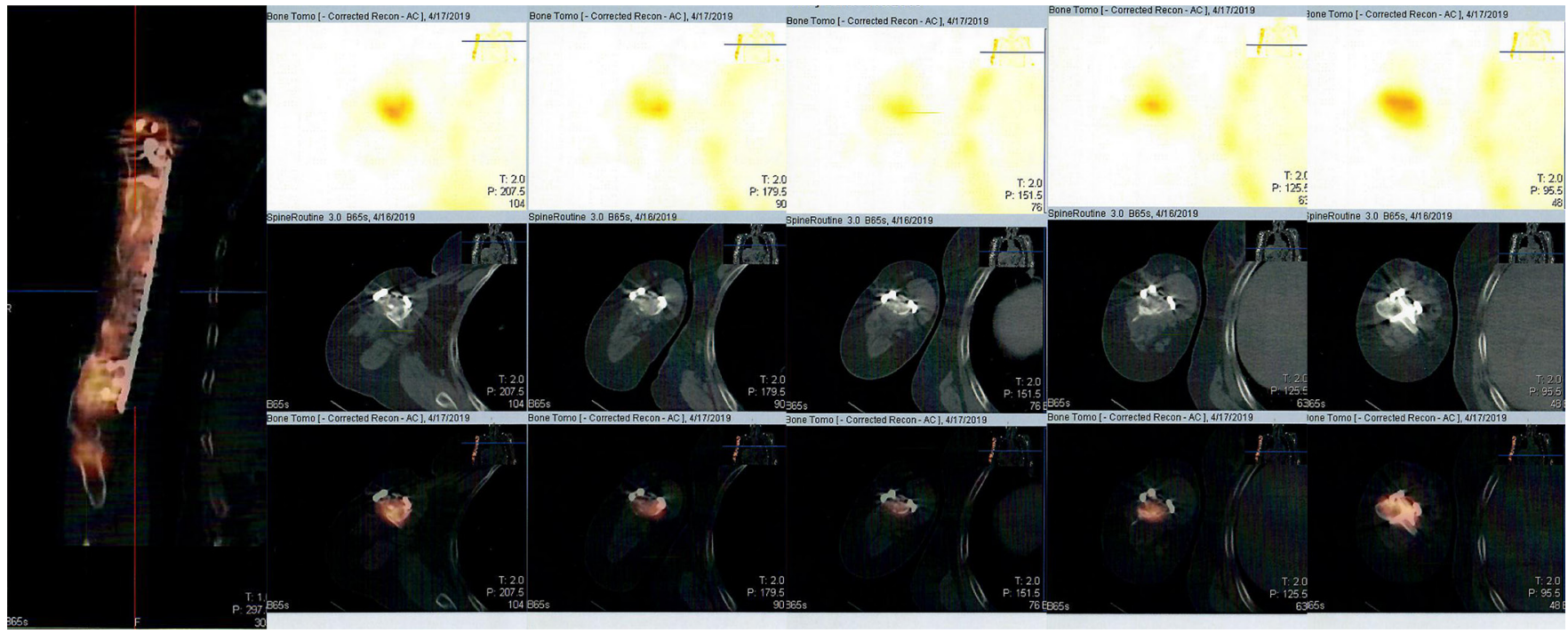

Figure 5 ECT/CT scans of a patient with large infected bone defect in the humerus. Scans at 9 months after operaion revealed bonetissue changes in the right humerus accompanied by active bone metabolism. Radioactive materials were uneven, highly distributed, and concentrated.

\section{Discussion}

Thus far, the induced membrane technique has shown excellent prospects in large infected bone defect reconstruction due to the advantages of simple operation procedure, straightforward postoperative nursing, shorter treatment duration (compared with the Ilizarov technique), and faster recovery of limb function (27-30). For the first time, we chose the antibiotic-loaded CS/CP composite as the supplement to autologous bone graft during the reconstruction stage of the induced membrane technique to overcome the significant demand for autologous bone and lack of sustained-release antibiotics.

In this study, after 3 months, both primitive callus formation and bone substitute absorption could observed. The average duration for full corticalization was around 29 weeks, and the union rate of all the 12 cases was $100 \%$. Bone consolidation with corticalization was regularly observed within 4 months through the traditional induced membrane technique in which only autologous bone graft was used in the reconstruction stage (31). Thus, compared with the traditional technique, our technique might have a longer duration of union time; however, all cases obtained a successful union. We noticed that in most cases, at 9 months, the remolding was finished, and the new bone density was the same as the surrounding bone tissue. What is intriguing is that in the humeral defect case, at 9 months, ECT examination was still positive, indicating that the osteogenesis metabolism might still have been present. The detailed osteogenesis process in our method still requires further investigation. We did not find a significant relationship between union time and defect size. Masquelet also found corticalization of the reconstructed bone segment is independent of the initial size of the defect (31).

In our study, the ratio of CS/CP composite supplement and autologous bone graft was 1:3. Masquelet surmised that isolated cancellous allograft or bone substitute inside the membrane is not sufficient for obtaining full bone formation because allograft or bone substitute does not contain stem cells or growth factors. Bone substitutes can be associated with cancellous autologous bone with a ratio which should not exceed 1:3. There have been some reports of extenders, including cancellous allograft or demineralized bone matrix, being used in patients when the autologous bone graft was insufficient $(32,33)$. In these reports, the ratio of extender and auto bone graft did not exceed 1:3, and there was no increase in complication or nonunion rates. However, allografts may increase the risk of disease transmission and infection.

However, Gupta et al. and Sasaki et al. attempted to use $\beta$-TCP as an extender by grafting autologous cancellous bone and $\beta$-TCP mixed in approximately the same proportions (1:1). Most patients achieved satisfactory 
healing results, even though $\beta$-TCP does not have osteoinductive ability $(34,35)$. Therefore, the appropriate ratio of bone substitute to auto cancellous bone still needs to be explored.

Some studies report failures of bone reconstruction through the induced membrane technique due to recurrent infection. The traditional induced membrane induced technique had inadequte anti-infection efficiency, and it can only be used once the infection is definitively eradicated. As we know, thorough debridement combined with systemic and local administration of antibiotics is a widely accepted treatment strategy against osteomyelitis $(36,37)$. The ability of biomaterials to locally deliver antibiotics has greatly contributed the anti-infection efficacy (20).

Earlier studies have shown that the antibiotic elution of calcium sulfate alone has a marked decrease in concentration within the initial 3 days (38). However, elution of CP calcium phosphate can be prolonged to 8 weeks (39). When the CS and CP were combined with composites, the antibiotic elution was around 4 weeks (40). Scharer and Sanicola found that the CS/CP composite, when combined with vancomycin, elutes vancomycin in a gradual and sustained fashion for 22 days in vitro with a ratio of $1 \mathrm{~g} /$ to $10 \mathrm{~g}$ (41). Furthermore animal studies have discovered that the CS/CP composite loaded with vancomycin ( $2 \mathrm{~g} / 20 \mathrm{~g})$ could effectively prevent osteomyelitis and enhance bone defect healing through improved bone remodeling $(20,38)$. The local elution ability of the CS/CP composite enables the local antibiotic concentration to be 100-200 times over intravenous dose (42). In our previous study, vancomycin $300 \mathrm{mg} / \mathrm{cc}$ was loaded with CS/CP to treat focal infected bone defect, and demonstrated excellent anti-infection efficacy (43). Thus, the concentration of vancomycin $300 \mathrm{mg} / \mathrm{cc}$ loaded with CS/CP combined with the induced membrane technology might be effective.

This is one of the reasons why we introduced the CS/ $\mathrm{CP}$ composite to the induced membrane technique as an expander; theoretically, we think that the antibiotic loading ability can enhance anti-infection efficiency to combat residual latent infection. In our study, all 12 cases of bone defect were accompanied by infection, and no cases showed signs of recurrence in the latest follow-up, even though 2 cases had exudation from the wound, which did not show a relation with relapse. Cases with complications were resolved by dressing changes.

The choice of antibiotic must take into consideration local microbial causative organisms, as well as the notion that high concentrations of certain antibiotics can affect the normal bone regeneration process (44). The reason we chose vancomycin as the local antibiotic in our study can be explained as follows: firstly, the water-soluble vancomycin has a dose-dependent nature. Secondly, vancomycin is effective against the most common causative organisms in osteomyelitis, particularly methicillin-resistant Staphylococcus aureus (MRSA) and other Staphylococci bacteria (45-48). The microbial culture showed that the most common bacteria in this study were Staphylococcus (5 out of 12), all of which were sensitive to vancomycin. Thirdly, high concentrations of vancomycin will not affect the normal bone regeneration process.

Moreover, through appropriate postoperative functional exercise guided by our professional physician, all patients recovered well after surgery and were able to engage in necessary daily activities, which greatly improved their quality of life. However, in our study, the total weight bearing was not allowed for 6 to 7 months, until the satisfactory corticalization was present. Masquelet indicated that for the induced membrane technique, a period of 3-4 months is too short to allow total weight bearing; instead, the recovery of weight bearing should be gradually acquired within 6-7 months after the definite reconstruction, regardless of surgical stabilization (31).

\section{Conclusions}

In summary, the CS/CP composite demonstrated capabilities of biodegradation, antibiotic eluding, and osteogenesis. For the first time, this study provided evidence supporting CS/CP as an effective and safe bone graft expander in the induced membrane technique for treating large infected bone defect. This technique is especially useful for patients who have previously undergone autologous bone grafting and for young or elderly patients who have limited sources of autologous bone or low osteogenic capacity. In addition, this technique may help decrease the infection recurrence resulting from the continuous local elution of antibiotics.

There are certain shortcomings to our study. Our research was a retrospective clinical study with a small number of cases. In the future, a randomized controlled trial involving a larger patient sample should be performed, and the traditional treatment of autologous bone graft alone should be used as the control. In our study, the average follow-up period was 16 months, and a longer period of follow-up will help confirm the usefulness of the $\mathrm{CS} / \mathrm{CP}$ composites expander in the induced membrane 
technique. While we used a 3:1 ratio of autologous bone to $\mathrm{CS} / \mathrm{CP}$ composite expander, the optimal ratio of the $\mathrm{CS} / \mathrm{CP}$ composite and autologous bone and concentration of antibiotic load should be further explored to ensure osteogenic and anti-infection efficacy.

\section{Acknowledgments}

Funding: This study was supported by the Innovation Development Fund Project of Tangdu Hospital (No. TC201903 to YZ) and the Military Medical Science and Technology Youth Training Program (No. 18QNP022 to YZ).

\section{Footnote}

Reporting Checklist: The authors have completed the STROBE reporting checklist. Available at http://dx.doi. org/10.21037/atm-20-1932

Data Sharing Statement: Available at http://dx.doi. org/10.21037/atm-20-1932

Conflicts of Interest: All authors have completed the ICMJE uniform disclosure form (available at http://dx.doi. org/10.21037/atm-20-1932). The authors have no conflicts of interest to declare.

Ethical statement: The authors are accountable for all aspects of the work in ensuring that questions related to the accuracy or integrity of any part of the work are appropriately investigated and resolved. The study was conducted in accordance with the Declaration of Helsinki (as revised in 2013). The study was approved by the institutional ethics board of Tang Du Hospital, Fourth Military Medical University (no. TDLL-201709-19). All patients provided informed consent preoperatively.

Open Access Statement: This is an Open Access article distributed in accordance with the Creative Commons Attribution-NonCommercial-NoDerivs 4.0 International License (CC BY-NC-ND 4.0), which permits the noncommercial replication and distribution of the article with the strict proviso that no changes or edits are made and the original work is properly cited (including links to both the formal publication through the relevant DOI and the license). See: https://creativecommons.org/licenses/by-nc$\mathrm{nd} / 4.0 \%$.

\section{References}

1. Lasanianos NG, Kanakaris NK, Giannoudis PV. Current management of long bone large segmental defects. Orthop Trauma 2010;24:149-63.

2. Gugala Z, Gogolewski S. Regeneration of segmental diaphyseal defects in sheep tibiae using resorbable polymeric membranes: a preliminary study. J Orthop Trauma 1999;13:187-95.

3. Masquelet AC, Fitoussi F, Begue T, et al. Reconstruction of the long bones by the induced membrane and spongy autograft. Ann Chir Plast Esthet 2000;45:346-53.

4. Aho OM, Lehenkari P, Ristiniemi J, et al. The mechanism of action of induced membranes in bone repair. J Bone Joint Surg Am 2013;95:597-604.

5. Gouron R, Petit L, Boudot C, et al. Osteoclasts and their precursors are present in the induced-membrane during bone reconstruction using the Masquelet technique. J Tissue Eng Regen Med 2017;11:382-9.

6. Christou C, Oliver RA, Yu Y, et al. The Masquelet technique for membrane induction and the healing of ovine critical sized segmental defects. PLoS One 2014;9:e114122.

7. Betz RR. Limitations of autograft and allograft: New synthetic solutions. Orthopedics 2002;25:s561-70.

8. Tilkeridis K, Touzopoulos P, Ververidis A, et al. Use of demineralized bone matrix in spinal fusion. World $\mathrm{J}$ Orthop 2014;5:30-7.

9. Gunzburg R, Szpalski M, Passuti N, et al. The use of bone substitutes in spine surgery: a state of the art review. Springer Science \& Business Media, 2002.

10. Zimmermann G, Moghaddam A. Allograft bone matrix versus synthetic bone graft substitutes. Injury 2011;42:S16-21.

11. Khan SN, Cammisa FPJ, Sandhu HS, et al. The biology of bone grafting. J Am Acad Orthop Surg 2005;13:77-86.

12. Evaniew N, Tan V, Parasu N, et al. Use of a calcium sulfate-calcium phosphate synthetic bone graft composite in the surgical management of primary bone tumors. Orthopedics 2013;36:e216-22.

13. Zhang J, Liu WZ, Schnitzler V, et al. Calcium phosphate cements for bone substitution: chemistry, handling and mechanical properties. Acta Biomater 2014;10:1035-49.

14. Ginebra MP, Espanol M, Montufar EB, et al. New processing approaches in calcium phosphate cements and their applications in regenerative medicine. Acta Biomater 2010;6:2863-73.

15. Bucholz RW. Nonallograft osteoconductive bone graft 
substitutes. Clin Orthop Relat Res 2002;(395):44-52.

16. Urban RM, Turner TM, Hall DJ, et al. Increased bone formation using calcium sulfate-calcium phosphate composite graft. Clin Orthop Relat Res 2007;459:110-7.

17. Roberts TT, Rosenbaum AJ. Bone grafts, bone substitutes and orthobiologics: the bridge between basic science and clinical advancements in fracture healing. Organogenesis 2012;8:114-24.

18. Fillingham YA, Cvetanovich GL, Haughom BD, et al. Bioceramic bone graft substitute for treatment of unicameral bone cysts. J Orthop Surg (Hong Kong) 2016;24:222-7.

19. Tan V, Evaniew N, Finlay K, et al. Chronology of the radiographic appearances of the calcium sulfatecalcium phosphate synthetic bone graft composite following resection of bone tumors: A follow-up study of postoperative appearances. Can Assoc Radiol J 2016;67:21-7.

20. Boyle KK, Sosa B, Osagie L, et al. Vancomycin-laden calcium phosphate-calcium sulfate composite allows bone formation in a rat infection model. PloS One 2019; 14:e0222034.

21. Landgraeber S, Warwas S, Claßen T, et al. Modifications to advanced core decompression for treatment of avascular necrosis of the femoral head. BMC Musculoskelet Disord 2017;18:479.

22. Zalavras CG, Patzakis MJ, Holtom P. Local antibiotic therapy in the treatment of open fractures and osteomyelitis. Clin Orthop Relat Res 2004;427:86-93.

23. Cook SD, Baffes GC, Wolfe MW, et al. The effect of recombinant human osteogenic protein-1 on healing of large segmental bone defects. J Bone Joint Surg Am 1994;76:827-38.

24. Salkeld SL, Patron LP, Barrack RL, et al. The effect of osteogenic protein-1 on the healing of segmental bone defects treated with autograft or allograft bone. J Bone Joint Surg 2001;83:803-16.

25. Paley D, Catagni MA, Argnani F, et al. Ilizarov treatment of tibial nonunions with bone loss. Clin Orthop Relat Res 1989;241:146-65.

26. Gandek B, Ware JE. SF-36 health Survey manual and interpretation guide. Boston: the Health Institute, New England Medical Center, 1993.

27. Tong K, Zhong Z, Peng Y, et al. Masquelet technique versus Ilizarov bone transport for reconstruction of lower extremity bone defects following post traumatic osteomyelitis. Injury 2017;48:1616-22.

28. Gao S, Tian S, Luo J, et al. Study of the therapy of multiple myeloma monoclonal antibody. Pak J Pharm Sci 2015;28:415-20.

29. Fan SD, Liu ZH, Hu WH, et al. Excision of necrotic and infected tissues combined with induced membrane and external fixator technique for the treatment of chronic osteomyelitis in tibia after fracture operation. Zhongguo Gu Shang 2017;30:372-6.

30. Mühlhäusser J, Winkler J, Babst R, et al. Infected tibia defect fractures treated with the Masquelet technique. Medicine (Baltimore) 2017;96:e6948.

31. Masquelet AC. Induced membrane technique: pearls and pitfalls. J Orthop Trauma 2017;31:S36-8.

32. Masquelet AC. Muscle reconstruction in reconstructive surgery: soft tissue repair and long bone reconstruction. Langenbecks Arch Surg 2003;388:344-6.

33. Masquelet AC, Begue T. The concept of induced membrane for reconstruction of long bone defects. Orthop Clin North Am 2010;41:27-37.

34. Gupta S, Malhotra A, Jindal R, et al. Role of beta tricalcium phosphate-based composite ceramic as bone-graft expander in Masquelet's-induced membrane technique. Indian J Orthop 2019;53:63.

35. Sasaki G, Watanabe Y, Miyamoto W, et al. Induced membrane technique using beta-tricalcium phosphate for reconstruction of femoral and tibial segmental bone loss due to infection: technical tips and preliminary clinical results. Int Orthop 2018;42:17-24.

36. Keating JF, Simpson A, Robinson CM. The management of fractures with bone loss. J Bone Joint Surg $\mathrm{Br}$ 2005;87:142-50.

37. Rohilla M, Saldanha K, Waseem M. Early versus delayed surgical treatment of open tibial fractures: effect on the rates of infection and need of secondary surgical procedures to promote bone union. Injury 2006;37:85-6.

38. Sanicola SM, Albert SF. The in vitro elution characteristics of vancomycin and tobramycin from calcium sulfate beads. J Foot Ankle Surg 2005;44:121-4.

39. Joosten U, Joist A, Frebel T, et al. Evaluation of an in situ setting injectable calcium phosphate as a new carrier material for gentamicin in the treatment of chronic osteomyelitis: studies in vitro and in vivo. Biomaterials 2004;25:4287-95.

40. Zelken J, Wanich T, Gardner M, et al. PMMA is superior to hydroxyapatite for colony reduction in induced osteomyelitis. Clin Orthop Relat Res 2007;462:190-4.

41. Scharer BM, Sanicola SM. The in vitro elution characteristics of vancomycin from calcium phosphatecalcium sulfate beads. J Foot Ankle Surg 2009;48:540-2. 
42. McPherson EJ, Dipane MV, Sherif SM. Dissolvable antibiotic beads in treatment of periprosthetic joint infection and revision arthroplasty-the use of synthetic pure calcium sulfate (Stimulan $®$ ) impregnated with vancomycin \& tobramycin. Joint Implant Surgery \& Research Foundation 2013;3:32-43.

43. Zhao Z, Wang G, Zhang Y, et al. The effect of calcium sulfate/calcium phosphate composite for the treatment of chronic osteomyelitis compared with calcium sulfate. Ann Palliat Med 2020;9:1821-33.

44. Hanssen AD. Local antibiotic delivery vehicles in the treatment of musculoskeletal infection. Clin Orthop Relat Res 2005;437:91-6.

45. McKee MD, Li-Bland EA, Wild LM, et al. A prospective, randomized clinical trial comparing an antibioticimpregnated bioabsorbable bone substitute with standard antibiotic-impregnated cement beads in the treatment of

Cite this article as: Zhao Z, Wang G, Zhang Y, Luo W, Liu S, Zeng Z, Liu Y, Zhou Y, Zhang Y. Induced membrane technique combined with antibiotic-loaded calcium sulfate-calcium phosphate composite as bone graft expander for the treatment of large infected bone defects: preliminary results of 12 cases. Ann Transl Med 2020;8(17):1081. doi: 10.21037/atm-20-1932 chronic osteomyelitis and infected nonunion. J Orthop Trauma 2010;24:483-90.

46. McKee MD, Wild LM, Schemitsch EH, et al. The use of an antibiotic-impregnated, osteoconductive, bioabsorbable bone substitute in the treatment of infected long bone defects: early results of a prospective trial. J Orthop Trauma 2002;16:622-7.

47. Turner TM, Urban RM, Gitelis S, et al. Radiographic and histologic assessment of calcium sulfate in experimental animal models and clinical use as a resorbable bone-graft substitute, a bone-graft expander, and a method for local antibiotic delivery: one institution's experience. J Bone Joint Surg Am 2001;83-A Suppl 2:8-18.

48. Urban RM, Turner TM, Hall DJ, et al. Healing of large defects treated with calcium sulfate pellets containing demineralized bone matrix particles. Orthopedics 2003;26:s581-5. 